\title{
Subwavelength chiral surface plasmons that carry tuneable orbital angular momentum
}

\author{
Felix Rüting,,$^{1, *}$ A. I. Fernández-Domínguez, ${ }^{2}$ L. Martín-Moreno, ${ }^{3}$ and F. J. García-Vidal ${ }^{1, \dagger}$ \\ ${ }^{1}$ Departamento de Física Teórica de la Materia Condensada, Universidad Autónoma de Madrid, Madrid E-28049, Spain \\ ${ }^{2}$ Department of Physics, Imperial College London, London SW7 2AZ, United Kingdom \\ ${ }^{3}$ Instituto de Ciencia de Materiales de Aragón and Departamento de Física de la Materia Condensada, \\ CSIC-Universidad de Zaragoza, Zaragoza E-50009, Spain
}

(Received 4 March 2012; revised manuscript received 17 July 2012; published 16 August 2012)

\begin{abstract}
We show that a helically grooved metal wire supports chiral surface plasmon polaritons (SPPs) that carry nonzero orbital angular momentum (OAM). This OAM can be tuned to have integer or fractional values by adjusting the mode wave vector. The dispersion relation and angular characteristics of the chiral modes are determined numerically and explained with the help of an effective mode index. Chiral SPPs offer the possibility to control both the chirality and the OAM of electromagnetic fields at the subwavelength scale.
\end{abstract}

DOI: $10.1103 /$ PhysRevB.86.075437

PACS number(s): 42.25.Bs, 42.50.Tx, 73.20.Mf

\section{INTRODUCTION}

During the last few years there have been several fundamental studies on electromagnetic (EM) metamaterials that present structural chirality (for a recent review see Ref. 1), leading to the emergence of interesting phenomena like asymmetric light propagation ${ }^{2}$ and negative refraction. ${ }^{3-5}$ Within a plasmonic perspective, it has been also shown that a carefully chosen superposition of three surface plasmon polaritons (SPPs) propagating on a metallic nanowire can be used to devise a chiral SPP. $^{6}$ In principle, due to their localized nature, SPPs could add subwavelength confinement to the chiral characteristics.

On the other hand, the fact that circular polarized light carries angular momentum was known since the early works of Maxwell and already demonstrated experimentally in 1936. ${ }^{7}$ However, interest in the angular momentum of light was reawakened in 1992 when it was demonstrated ${ }^{8}$ that Laguerre-Gaussian beams can transport orbital angular momentum (OAM) ${ }^{9-12}$ The OAM of EM fields has been also studied using plasmonic vortex structures, ${ }^{13,14}$ and it has been extensively used in research areas as diverse as biophysics, micromechanics, and microfluidics. ${ }^{15}$ At a quantum level, single photons with OAM have the potential for realizing highdimensional quantum spaces with applications in information technologies. ${ }^{16}$ Normally, OAM is associated with EM modes that are spatially extended. For many fundamental studies and practical applications, it would be desirable to work with localized light that could carry OAM at the subwavelength scale.

Here, we present a plasmonic structure that supports EM modes that are subwavelength confined and show the two aforementioned properties (chirality and OAM): a metal wire corrugated with a helical groove. Importantly, the length scale of the corrugation is much smaller than the wavelength so the system behaves as a metamaterial, where diffraction effects can be discarded. We present a detailed analysis of the chiral character and angular momentum properties of these surface EM modes and we also demonstrate two possible ways to excite them.

\section{FUNDAMENTAL PROPERTIES OF CHIRAL SURFACE PLASMONS}

In the inset of Fig. 1 we render a sketch of the helically grooved wire with a definition of the geometrical parameters: the radius of the cylinder $R$, the period of the array $\Lambda$, and the width $w$ and the depth $h$ of the groove. To present a general study of the chiral SPPs supported by this structure, we assume first that the metal behaves as a perfect electrical conductor (PEC) and show later in this section that the fundamental properties remain unchanged if a realistic metal in the optical regime is considered.

By using a PEC wire, the emergence of chiral SPPs in different frequency regimes could be analyzed by just scaling appropriately the geometrical parameters. In our calculations we will use the pitch $\Lambda$ as the unit length. Note that, as we work within the PEC approximation, the surface EM modes supported by this structure have a geometrical origin and can be considered as a special type of spoof SPPs. ${ }^{17,18}$ A previous experimental study of the surface EM modes supported by helically grooved metal wires in the terahertz regime was performed ${ }^{19}$ but no attention was paid to the chiral character and the OAM of these modes.

In Fig. 1 the dispersion relations of the different surface EM modes supported by the helically grooved wire are displayed. These bands have been computed using a finiteelement discretization of Maxwell equations. ${ }^{20}$ Notice that, in contrast to the chiral SPPs discussed in Ref. 6, the modal wavelength of the modes shown in Fig. 1 is always smaller than the free-space wavelength $\left(k_{z}>2 \pi / \lambda\right)$, which results in subwavelength confinement. Using the notation introduced for chiral metamaterials, ${ }^{3}$ we can characterize the surface EM modes by two labels: (i) an index $l$, related to the azimuthal dependence, which is determined by the number of azimuthal nodes $(2 l)$ that the EM fields present for small values of $k_{z}$; (ii) a sign (+ or -$)$ depending on the rotation direction at which the mode accumulates phase, + for a clockwise rotation and for the counterclockwise one (as usual, the rotation direction is determined in the propagation direction). The chirality of the SPP modes is illustrated by the fact that for positive and negative values of $k_{z}$, the following two relations hold:

$$
\begin{aligned}
& \omega_{ \pm}^{(l)}\left(k_{z}\right) \neq \omega_{ \pm}^{(l)}\left(-k_{z}\right) \\
& \omega_{ \pm}^{(l)}\left(k_{z}\right)=\omega_{\mp}^{(l)}\left(-k_{z}\right) .
\end{aligned}
$$

Equations (1) and (2) state that by changing the propagation direction of the EM mode, its rotation direction has to be also changed to keep the mode frequency fixed. This behavior 


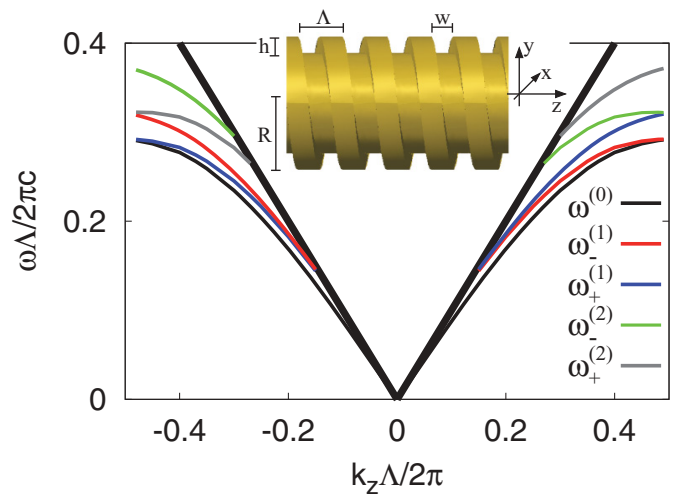

FIG. 1. (Color online) Inset: Sketch of a helically grooved wire with a definition of the different geometrical parameters $R, \Lambda, w$, and $h$ and of the three axes. Main panel: Dispersion relation of the surface EM modes supported by this structure with $R=1.5 \Lambda, w=0.5 \Lambda$, and $h=3 / 8 \Lambda$.

is distinctive of helically grooved metal wires, and it is not present in either noncorrugated metal wires, slanted grooves, ${ }^{21}$ or corrugated wires with a periodic array of rings. ${ }^{22}$ This is a direct consequence of the structural chirality of the grooved wire.

The results displayed in Fig. 1 also show that, in addition to the chiral degeneracy characterized by Eq. (2), a new type of degeneracy emerges at the band edges, $k_{z}= \pm \pi / \Lambda$ :

$$
\omega_{ \pm}^{(l)}( \pm \pi / \Lambda)=\omega_{\mp}^{(l+1)}( \pm \pi / \Lambda) .
$$

It is convenient to simplify the notation and combine the two labels into a mode index $m= \pm l$, in which the sign is related to the rotation direction and $l$ is the azimuthal index. The evolution of the EM fields associated with the first three chiral SPPs $(m=0, m= \pm 1)$ as their wave vector is increased from 0 to $\pi / \Lambda$ is analyzed in Fig. 2(a). To better illustrate the modal shape, in Fig. 2 we render the modulus of the real part of the $E$-field averaged along the $z$ direction over one unit cell. Notice that the wavefront is helical within each unit cell as it follows the shape of the groove. For small values of the wave vector $\left(k_{z}=0.3 \pi / \Lambda\right.$, upper row), the $E$-field patterns of the chiral SPP modes with $m=-1$ and $m=+1$ are very similar. However, for $k_{z}$ close to the band edge $\left(k_{z}=0.98 \pi / \Lambda\right.$, lower row), the modes with $m=0$ and $m=-1$ show the same azimuthal dependence, as expected from Eq. (3). The $E$ fields of the modes $m=-2,-3,-4$ evaluated at a wave vector close to the band edge $\left(k_{z}=0.98 \pi / \Lambda\right)$ are displayed in Fig. 2(b). Again, the $E$-field pattern of the mode $m=-2$ closely resembles that of the $m=1$ mode, but with an opposite rotation direction.

The degeneracy at the band edges introduced by the helical groove can be explained by the structure of the EM fields in a helical geometry. In such geometry, it is expected that all the field components follow the helical symmetry of the underlying physical system and hence they can be expanded as [with the cylindrical coordinates $(r, \phi, z)]^{23}$

$$
F_{m}(r, \phi, z)=A_{m}(r, z) e^{i\left(m+k_{z} \frac{\Lambda}{2 \pi}\right) \phi},
$$

with $A_{m}$ obeying Bloch's theorem in the $z$ direction. Therefore, the $\phi$ dependence of the fields is governed by an effective (a)
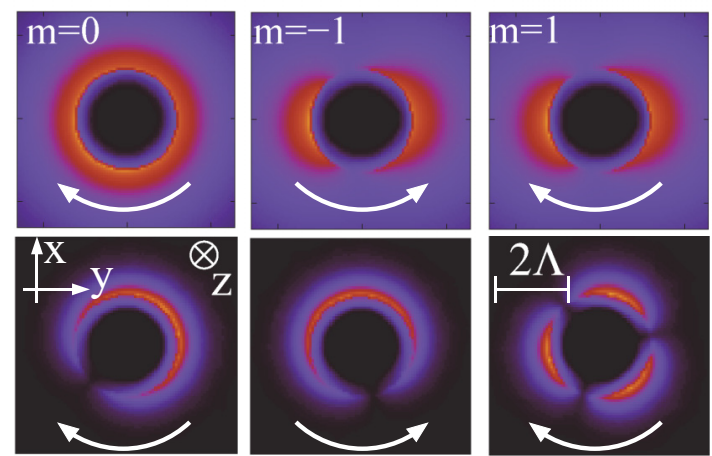

(b)

(c)
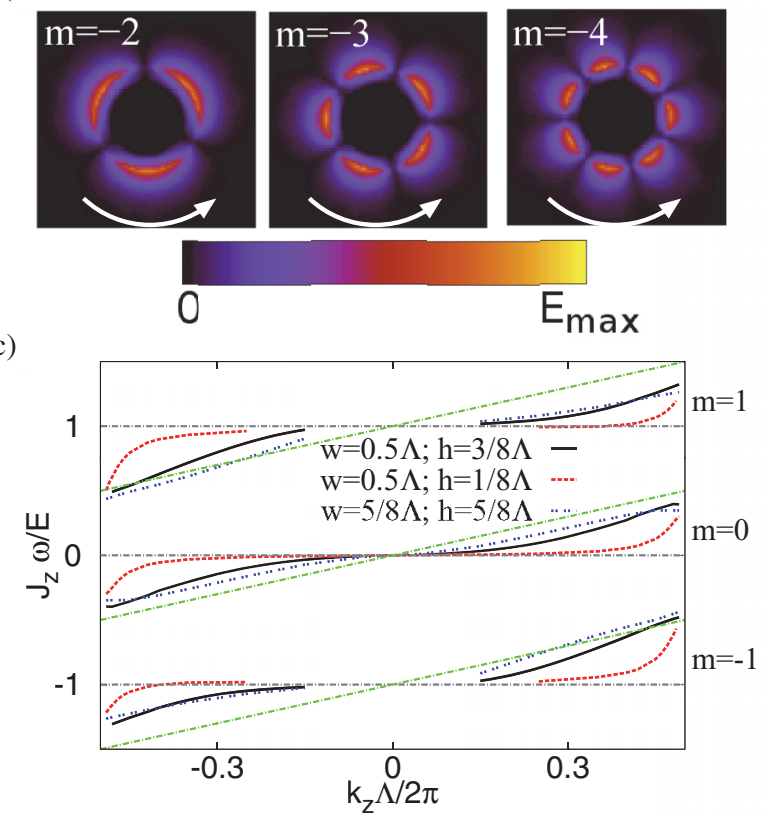

FIG. 2. (Color online) Modulus of the real part of the $E$ field averaged over one unit cell for (a) modes $m=0, m= \pm 1$ and (b) modes $m=-2,-3,-4$. Colors scale the field intensity from minimum (black) to maximum (yellow). The upper (lower) row of panel (a) corresponds to $E$ fields evaluated at $k_{z}=0.30 \pi / \Lambda$ $\left(k_{z}=0.98 \pi / \Lambda\right)$. The $E$ fields in panel (b) are computed also at $k_{z}=0.98 \pi / \Lambda$. The white arrows show the rotation direction of the chiral SPPs. As in Fig. 1, the values of the geometrical parameters are $R=1.5 \Lambda, w=0.5 \Lambda$, and $h=3 / 8 \Lambda$, and the shown orientation of the axes and the scale bar are valid for all crosscuts. (c) Evolution of $J_{z} \omega / E$ with $k_{z}$ for the first three modes calculated for a fixed $R$, $R=1.5 \Lambda$, and three different sets of $(w, h)$ values. Dotted green lines render the behavior of the effective mode index $\tilde{m}$.

mode index $\tilde{m}\left(k_{z}\right)$, accounting for the phase accumulated while moving along one period of the helix

$$
\tilde{m}\left(k_{z}\right)=m+k_{z} \frac{\Lambda}{2 \pi} .
$$

This simple relation explains the degeneracy in Eq. (3) and accounts for the evolution of the $E$-field profiles with the wave vector. For small values of $k_{z}, \tilde{m} \approx m$, and the number of nodes is always even. Close to the band edge, $k_{z}=\pi / \Lambda$, $\tilde{m} \approx m+1 / 2$, implying that the number of azimuthal nodes is odd for all values of $m$, as observed in Figs. 2(a) and 2(b). 
A helical wavefront, as that associated with a chiral SPP, is related to the OAM of the EM field. ${ }^{24-29}$ The fact that the effective mode index $\tilde{m}$ may exhibit noninteger values also suggests that the chiral SPPs supported by the corrugated wire could carry fractional angular momentum. To test this hypothesis, we quantify the OAM associated with the different chiral SPPs by evaluating the $z$ component of the angular momentum density ${ }^{24}$ integrated over a plane perpendicular to the wire axis

$$
J_{z}=\frac{1}{2} \epsilon_{0} \int r d r \int d \phi[\mathbf{r} \times \operatorname{Re}(\mathbf{E} \times \overline{\mathbf{B}})]_{z} .
$$

Following the case of Laguerre-Gaussian beams, ${ }^{8}$ we normalize $J_{z}$ to $E / \omega$, with $E$ being the energy density integrated over the same plane. In Fig. 2(c), we render the evolution of $J_{z} \omega / E$ with $k_{z}$ of the first three chiral SPP modes $(m=0, \pm 1)$ and for three different sets of geometrical parameters. For small values of $k_{z}$ this magnitude is governed by $m$, resulting in integer values for the normalized OAM. However, when $k_{z}$ approaches the band edges, $J_{z}$ closely follows the effective mode index $\tilde{m}$, implying that the chiral SPPs can exhibit fractional OAM near the band edges. Note that although the evolution with $k_{z}$ depends on the particular values of the geometrical parameters, the normalized OAMs at $k_{z} \approx \pm \pi / \Lambda$ are close to $\pm 1 / 2, \pm 3 / 2$.

The chirality of the modes, as expressed by Eqs. (1) and (2) and shown by the dispersion relation in Fig. 1, also results in an asymmetric transport behavior of the grooved wire. This is exemplified in Fig. 3, where the propagation of the mode with $m=-1$ along a wire with a varying groove depth is shown [see Fig. 3(b) for the groove profile used]. The frequency $\left(\omega=0.32 \frac{2 \pi c}{\Lambda}\right)$ is chosen such that the selected mode is supported by a grooved wire with a depth of the groove similar to the depth at the ends of the system. While for a wire with the deepest groove [central part of the system; see Fig. 3(b)] the mode with $m=-1$ only exists for $k_{z}<0$. The asymmetric transport behavior is clearly visible both in the

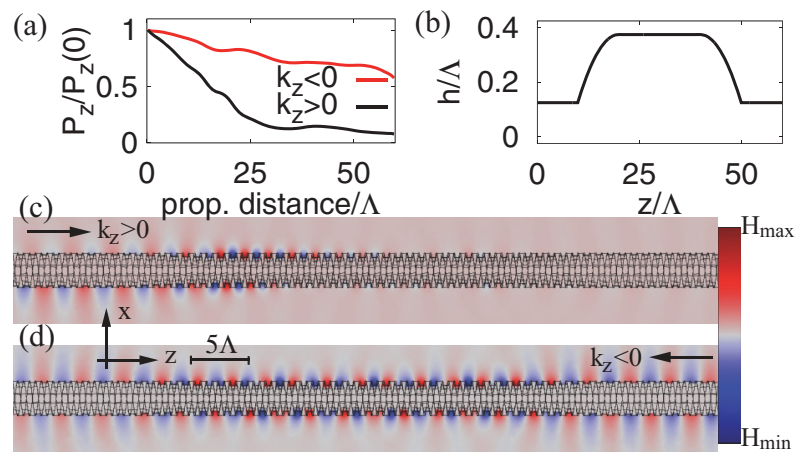

FIG. 3. (Color online) Asymmetric transport behavior along a wire with a varying groove depth. (a) $z$ component of the Poynting vector along the wire integrated over planes perpendicular to the wire axis and normalized to the Poynting vector at the input end. (Note that the propagated distance is measured in the positive $z$ direction for $k_{z}>0$ and in the negative $z$ direction for $k_{z}<0$ ). (b) Groove profile used. Real part of $H_{\phi}$ along the wire for (c) $k_{z}>0$ and (d) $k_{z}<0$. The color scale ranges from blue (minimum) to red (maximum) and the maximum field intensity in (c) is roughly twice as big as the one in (d). normalized Poynting vector along the wire [Fig. 3(a)] and in the field plots [Fig. 3(c)]. Therefore, by a proper design of the geometrical parameters and by an appropriately chosen operating wavelength, the asymmetric transport behavior of the grooved wire can be explored for a selected mode. Nevertheless, the asymmetric transport behavior should be not confused with one-way optical elements such as optical isolators ${ }^{30-32}$ and it should be kept in mind that the presented system is reciprocal. So the transport behavior for the mode with $m=1$ would be the opposite (propagation for $k_{z}>0$ and suppression for $k_{z}<0$ ).

So far we have described the dielectric properties of the wire within the PEC approximation. However, the dispersive behavior of a realistic metal and the related losses become relevant in the optical regime. To demonstrate that the fundamental properties of the chiral SPPs are present in this regime, we study the modes of a gold wire with a pitch of $\Lambda=160 \mathrm{~nm}$. The dielectric properties of Au are modeled from the fitting to Johnson and Christy experimental data. ${ }^{34}$ The dispersion relations of the corresponding modes are shown in Fig. 4, and the same qualitative behavior as the one for a PEC wire (see Fig. 1) is found. Unlike to the PEC case, absorption losses are now significant and hence the propagation length $L$ becomes of interest. It is obtained from the imaginary part of the eigenfrequencies ( $L=\frac{2 \pi v_{g}}{\operatorname{Im}(\omega)}$ with the group velocity $\left.v_{g}=\frac{d \omega}{d k_{z}}\right)$ and shown in the inset of Fig. 4. The propagation length for the different chiral SPPs are reasonably large, and hence the fundamental properties of the chiral SPPs could be explored even in the optical regime using conventional noble metals.

\section{WAYS OF EXCITATION}

In the following, we present two ways of exciting a selected chiral SPP characterized by a given OAM and supported again by a PEC wire. A particular SPP mode can be excited by starting with a mode of a noncorrugated wire and by gradually introducing the groove along the wire. To demonstrate this strategy, we use a wire which is noncorrugated in the input end, and where the depth of the groove is increased from $h=0$ to $h_{\max }=0.375 \Lambda$ [the depth profile used is shown in

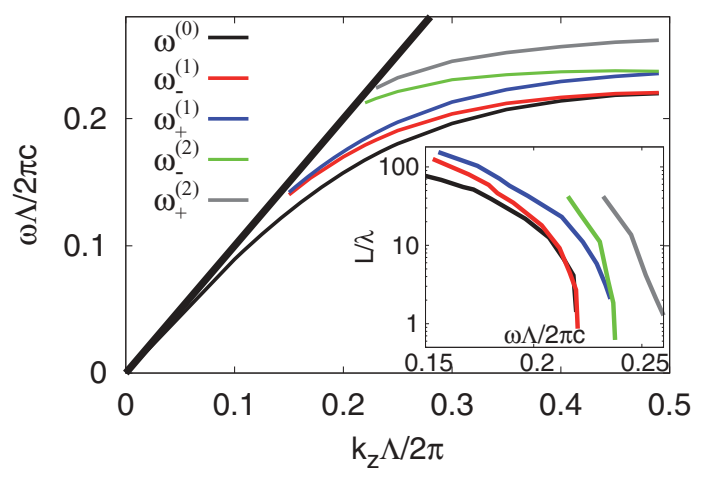

FIG. 4. (Color online) Dispersion relation of the chiral plasmons supported by a gold wire with $\Lambda=160 \mathrm{~nm}, R=240 \mathrm{~nm}, w=80 \mathrm{~nm}$, and $h=60 \mathrm{~nm}$. The inset shows the propagation lengths calculated via the imaginary part of the eigenfrequency (notice the logarithmic scale). 


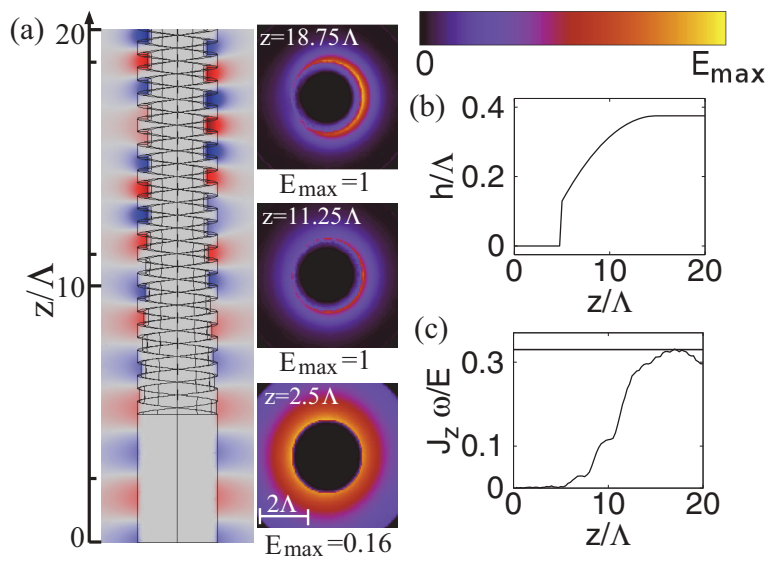

FIG. 5. (Color online) Control of the EM field pattern and OAM of a chiral SPP by changing adiabatically the depth of the groove. (a) Real part of $H_{\phi}$ evaluated at the $y=0$ plane and $x y$ crosscuts showing the modulus of the real part of the $E$ field (integrated over one period of the helical groove) at different $z$ positions along the wire. For each crosscut the color scale used is adapted to the respective field intensities and the maximum intensity (normalized to 1 for large values of $z$ ) is indicated in each case. Panels (b) and (c) render the groove's depth profile used in our calculations and the $z$ component of the normalized OAM along the wire, respectively.

Fig. 5(b)]. At the input end, the fundamental mode $(m=0)$ of a noncorrugated wire ${ }^{33}$ is excited, and the frequency is chosen so that the modal wave vector is $k_{z}=0.44 \pi / \Lambda$ for $h_{\max }$. The plot of the real part of $H_{\phi}$ (the dominant magnetic component of the fundamental mode) in Fig. 5(a) shows that, as the depth of the groove increases, the chiral SPP becomes bounded more strongly to the wire (in agreement with an increasing value of $k_{z}$ ). Moreover, also the angular profile of the field changes from azimuthal independence to the one expected for a wave vector close to the band edge ( $\tilde{m}=0.5)$, see Fig. 2(a). The increasing groove depth also affects the normalized OAM [see Fig. 5(c)] and the value of $J_{z}$ at the end of the wire almost coincides with that corresponding to the eigenmode calculation. The decrease of $J_{z}$ at the very end of the wire is related to reflexions of the SPP at the boundary of the simulation volume. We stress that this calculation is presented here just for proof-of-principle purposes and no attempt to fully optimize the structure has been made. Notice that, despite the absence of optimization, around $70 \%$ of the energy of the fundamental mode of the noncorrugated wire is transferred to the OAM-carrier chiral SPP in a length scale that is only three times the SPP modal wavelength.

The way of exciting a desired mode discussed above requires a gradual change of the groove depth, which would require an accurate manufacture of the structure. An alternative way of exciting chiral SPPs in helically grooved wires is to place an emitter of subwavelength size (a quantum dot, for example) in the vicinity of the metal wire, in a way very much similar to what has been done to study the quantum nature of SPPs in noncorrugated metal wires. ${ }^{35}$ An important asset of this strategy is that it leads to the excitation of two SPPs propagating in opposite directions. Due to their chiral character, these SPP modes have different OAM ( $J_{z}$ of the same absolute value but different sign). We demonstrate the feasibility of this approach by analyzing the excitation of the modes with $m=2$ and $m=-2$ for the set of geometrical parameters used in Fig. 1. To this end, we simulate the emitter as a point dipole (oriented perpendicularly to the wire axis), placed at $y=R+0.125 \Lambda$ away from the wire axis. In Fig. 6(a) the normalized OAM along the wire is shown. After an initial region dominated by the dipole excitation, the normalized $J_{z}$ is in good agreement with the result of the eigenmode calculation [indicated by the horizontal lines in Fig. 6(a)]. Moreover the EM field patterns [see Fig. 6(b)] at the wire's ends show that the desired modes have been efficiently excited. Notice the opposite rotation directions of the two chiral SPPs

(a)

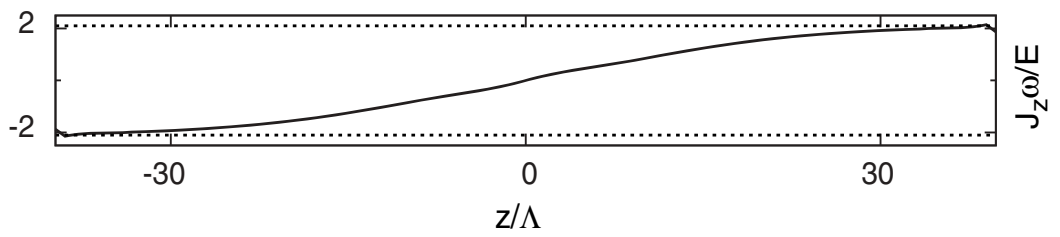

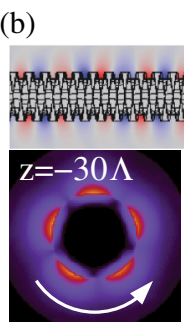

$\mathrm{E}_{\max }=0.18$

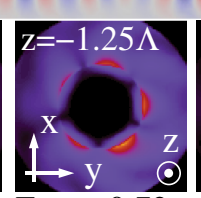

$\mathrm{E}_{\max }=0.72$

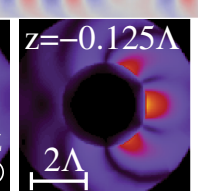

$\mathrm{E}_{\max }=1$ $\mathrm{z} / \Lambda$
$5 \Lambda$

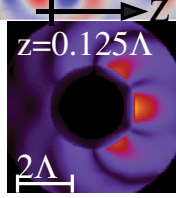

$\mathrm{E}_{\max }=1$

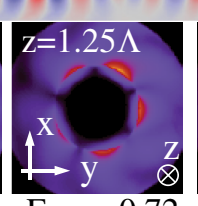

$\mathrm{E}_{\max }=0.72$

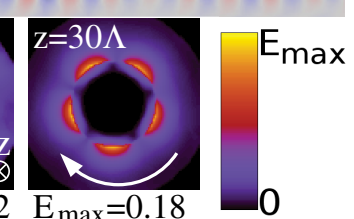

FIG. 6. (Color online) Excitation of two chiral SPPs by a point dipole. The set of geometrical parameters is the same as in Fig. $1: R=1.5 \Lambda$, $w=0.5 \Lambda$, and $h=3 / 8 \Lambda$. The dipole is located at $x=0, y=R+0.125 \Lambda$, and $z=0$ and the frequency is chosen such that for $k_{z}>0$ and for $k_{z}<0$ only one mode exists ( $m=2$ for $k_{z}>0$ and $m=-2$ for $k_{z}<0$, respectively). (a) Evolution of the normalized $J_{z}$ with $z$ (the horizontal dotted lines indicate the eigenmode results). (b) Real part of the dominant component of the magnetic field $\left(H_{\phi}\right)$ evaluated at the $(x, z)$ plane $y=0$ (upper panel) and $(x, y)$ crosscuts of the $E$ field computed at different $z$ 's along the wire. For each crosscut the color scale used is adapted to the respective field intensities and the maximum intensity (normalized to the one close to the dipole) is indicated The white arrows show the rotation direction of the two chiral SPPs. Observe the different orientation of the axes for $z>0$ and $z<0$. 
[as denoted by the white arrows in Fig. 6(b)], which also results in the different signs of $J_{z}$ along the two propagation directions.

\section{CONCLUSIONS}

In conclusion, we have demonstrated that a helically grooved metal wire supports the propagation of chiral SPPs. These modes carry OAM that is integer at the center of the first Brillouin zone and fractional at its edges. A model based on an effective mode index offers a simple way of understanding their dispersion relations and associated OAM. Furthermore, the structural chirality causes an asymmetric transport behavior for the different modes. Importantly, we have shown that the fundamental properties of the chiral SPPs remain unchanged for realistic metals in the optical regime. Two different approaches for exciting the chiral SPPs have been also discussed, exemplifying the broad range of field patterns achievable in the system studied. We believe that helically grooved metal wires and their chiral SPPs could help to study the response of molecules or nanoparticles to chiral EM fields and to analyze light-matter interactions that involve the orbital angular momentum exchange at a subwavelength scale.

\section{ACKNOWLEDGMENTS}

This work has been sponsored by the Spanish Ministry of Science and Innovation under Contract No. MAT200806609-02 and project Nanolight.es. F.R. acknowledges support from the Deutsche Akademie der Naturforscher Leopoldina under Grant No. LPDS 2009-52. This work has been partially supported by the European Research Council, Grant No. 290981 (PLASMONANOQUANTA). *felix.ruting@uam.es

$\dagger$ †j.garcia@uam.es

${ }^{1}$ C. M. Soukoulis and M. Wegener, Nature Photon. 5, 523 (2011).

${ }^{2}$ V. A. Fedotov, P. L. Mladyonov, S. L. Prosvirnin, A. V. Rogacheva,

Y. Chen, and N. I. Zheludev, Phys. Rev. Lett. 97, 167401 (2006).

${ }^{3}$ J. B. Pendry, Science 306, 1353 (2004).

${ }^{4}$ E. Plum, J. Zhou, J. Dong, V. A. Fedotov, T. Koschny, C. M. Soukoulis, and N. I. Zheludev, Phys. Rev. B 79, 035407 (2009).

${ }^{5}$ S. Zhang, Y.-S. Park, J. Li, X. Lu, W. Zhang, and X. Zhang, Phys. Rev. Lett. 102, 023901 (2009).

${ }^{6}$ S. Zhang, H. Wei, K. Bao, U. Håkanson, N. J. Halas, P. Nordlander, and H. Xu, Phys. Rev. Lett. 107, 096801 (2011).

${ }^{7}$ R. A. Beth, Phys. Rev. 48, 471 (1935); 50, 115 (1936).

${ }^{8}$ L. Allen, M. W. Beijersbergen, R. J. C. Spreeuw, and J. P. Woerdman, Phys. Rev. A 45, 8185 (1992).

${ }^{9}$ S. J. van Enk and G. Niehuis, J. Mod. Opt. 41, 963 (1994).

${ }^{10}$ S. M. Barnett, J. Opt. B 4, 7 (2001); K. Y. Bliokh, M. A. Alonso, E. A. Ostrovskaya, and A. Aiello, Phys. Rev. A 82, 063825 (2010).

${ }^{11}$ S. M. Barnett, J. Opt. 13, 064010 (2011).

${ }^{12}$ L. Marrucci, E. Karimi, S. Slussarenko, B. Piccirillo, E. Santamato, E. Nagali, and F. Sciarrino, J. Opt. 13, 064001 (2011).

${ }^{13}$ H. Kim, J. Park, S.-W. Cho, S.-Y. Lee, M. Kang, and B. Lee, Nano Lett. 10, 529 (2010).

${ }^{14}$ J. Trevino, H. Cao, and L. Dal Negro, Nano Lett. 11, 2008 (2011).

${ }^{15} \mathrm{~J}$. P. Torres and L. Torner, Twisted Photons: Applications of Light with Orbital Angular Momentum (Wiley-VCH, New York, 2011).

${ }^{16}$ G. Molina-Terriza, J. P. Torres, and L. Torner, Nat. Phys. 3, 305 (2007).

${ }^{17}$ J. B. Pendry, L. Martin-Moreno, and F. J. Garcia-Vidal, Science 305, 847 (2004).

${ }^{18}$ F. J. Garcia-Vidal, L. Martin-Moreno, and J. B. Pendry, J. Opt. A: Pure Appl. Opt. 7, 97 (2005).

${ }^{19}$ A. I. Fernandez-Dominguez, C. R. Williams, F. J. Garcia-Vidal, L. Martin-Moreno, S. R. Andrews, and S. A. Maier, Appl. Phys. Lett. 93, 141109 (2008).
${ }^{20}$ All numerical calculations were done with COMSOL v3.5a, and for the eigenmode calculations a three-dimensional unit cell with periodic boundary conditions was used.

${ }^{21}$ J. J. Wood, L. A. Tomlinson, O. Hess, S. A. Maier, and A. I. Fernández-Domínguez, Phys. Rev. B 85, 075441 (2012).

${ }^{22}$ S. A. Maier, S. R. Andrews, L. Martin-Moreno, and F. J. GarciaVidal, Phys. Rev. Lett. 97, 176805 (2006).

${ }^{23}$ R. A. Waldron, Q. J. Mech. Appl. Math. 11, 438 (1958); P. J. Crepeau and P. R. Mcisaac, Proc. IEEE 52, 33 (1964).

${ }^{24}$ M. Padgett and L. Allen, Contemp. Phys. 41, 275 (2000).

${ }^{25}$ A. T. O'Neil, I. MacVicar, L. Allen, and M. J. Padgett, Phys. Rev. Lett. 88, 053601 (2002).

${ }^{26}$ K. Y. Bliokh, A. Niv, V. Kleiner, and E. Hasman, Nature Phot. 2, 748 (2008).

${ }^{27}$ Y. Gorodetski, N. Shitrit, I. Bretner, V. Kleiner, and E. Hasman, Nano Lett. 9, 3016 (2009).

${ }^{28}$ S. M. Barnett, J. Mod. Opt. 57, 1339 (2010).

${ }^{29}$ E. Hasman, Nature Nanotech. 5, 563 (2010).

${ }^{30}$ L. Feng, M. Ayache, J. Huang, Y.-L. Xu, M.-H. Lu, Y.-F. Chen, Y. Fainman, and A. Scherer, Science 333, 729 (2011).

${ }^{31}$ S. Fan, R. Baets, A. Petrov, Z. Yu, J. D. Joannopoulos, W. Freude, A. Melloni, M. Popović, M. Vanwolleghem, D. Jalas, M. Eich, M. Krause, H. Renner, E. Brinkmeyer, and C. R. Doerr, Science 335, 38b (2012).

${ }^{32}$ L. Feng, M. Ayache, J. Huang, Y.-L. Xu, M.-H. Lu, Y.-F. Chen, Y. Fainman, and A. Scherer, Science 335, 38c (2012).

${ }^{33}$ As SPP modes are not supported by noncorrugated PEC wires, in this simulation we have used a finite $\epsilon(\epsilon=-100+i)$ for describing the metal response.

${ }^{34}$ P. B. Johnson and R. W. Christy, Phys. Rev. B 6, 4370 (1972).

${ }^{35}$ A. V. Akimov, A. Mukherjee, C. L. Yu, D. E. Chang, A. S. Zibrov, P. R. Hemmer, H. Park, and M. D. Lukin, Nature (London) 450, 402 (2007). 